Supplement of Biogeosciences, 18, 1407-1415, 2021 https://doi.org/10.5194/bg-18-1407-2021-supplement (C) Author(s) 2021. This work is distributed under the Creative Commons Attribution 4.0 License.

(c) (1)

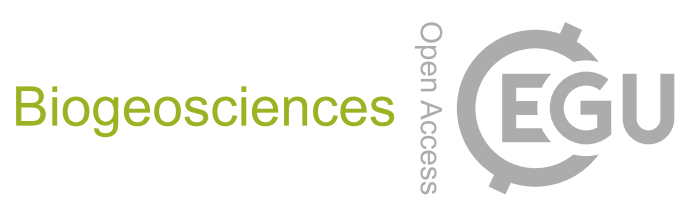

Supplement of

\title{
Technical note: Interpreting pH changes
}

Andrea J. Fassbender et al.

Correspondence to: Andrea J. Fassbender (andrea.j.fassbender@noaa.gov)

The copyright of individual parts of the supplement might differ from the CC BY 4.0 License. 


\section{S1. Determination of 2010 mean $\mathrm{pH}$ values}

Mean $\mathrm{pH}$ values provided in the supplemental information of Munro et al., (2015) were adjusted to the year 2010 using the $\mathrm{pH}$ trends and observing periods reported in Munro et al., (2015). The mean $\left[\mathrm{H}^{+}\right]$value for Station ALOHA provided in the main text of Dore et al. (2014) was adjusted to the year 2010 using the $\left[\mathrm{H}^{+}\right]$trend and observing period reported in Dore et al. (2014) and converted to $\mathrm{pH}$. Mean $\mathrm{pH}$ values were calculated for all other locations using (a) 12-month $\mathrm{pH}$ climatology data constructed from available time-series site data and referenced to the year 2010 or (b) a 2010-normalized, global $3^{\circ} \times 3^{\circ}$ gridded $\mathrm{pH}$ climatology based on Version 4 of the Surface Ocean $\mathrm{CO}_{2}$ Atlas (SOCAT-v4; Bakker et al., 2016), as described in Fassbender et al. (2017). The 2010 mean pH values are listed in Table S1. The 2010 mean $\left[\mathrm{H}^{+}\right]$values described in Text S2 were computed from 2010 mean pH values. All pH (and

$10\left[\mathrm{H}^{+}\right]$values are given at in situ temperatures.

\section{S2. Calculation of $\mathrm{pH}$ and $\left[\mathrm{H}^{+}\right]$trends and their uncertainties}

$\mathrm{pH}$ trends and uncertainties $(u)$ are provided in the main text of Bates et al. (2014; defined as one standard error of the slopes) and Sutton et al. (2014; defined as the measurement uncertainty propagated through a weighted linear regression) as well as in the supplemental information of Munro et al. (2015; defined as one standard error of the slopes). The $\left[\mathrm{H}^{+}\right]$trend and uncertainty (defined as one standard error of the slope) for Station ALOHA are provided in the main text of Dore et al. (2014) and were used along with the mean $\left[\mathrm{H}^{+}\right]$value (adjusted to 2010) to compute the $\mathrm{pH}$ trend and uncertainty. The two $p \mathrm{CO}_{2}$ trends and uncertainties given in the main text of Sutton et al. (2019) were converted to $\left[\mathrm{H}^{+}\right]$trends and uncertainties using the local, 2010-referenced, climatological (clim.) $d\left[\mathrm{H}^{+}\right] / d p \mathrm{CO}_{2}$ sensitivity derived from the SOCAT-v4 gridded data product described in Fassbender et al. (2017). That sensitivity is expected to change only marginally over the $21^{\text {st }}$ century (see Hagens and Middelburg, 2016). At each location considered, the $\left[\mathrm{H}^{+}\right]$trend and its uncertainty are given by:

$$
\begin{aligned}
& \left(\frac{d\left[\mathrm{H}^{+}\right]}{d t}\right)=\left(\frac{d\left[\mathrm{H}^{+}\right]}{d p C O_{2}}\right)_{c l i m .} \times\left(\frac{d p \mathrm{CO}_{2}}{d t}\right) \\
& u\left(\frac{d\left[\mathrm{H}^{+}\right]}{d t}\right)=\left(\frac{d\left[\mathrm{H}^{+}\right]}{d p \mathrm{CO}_{2}}\right)_{\text {clim. }} \times u\left(\frac{d p \mathrm{CO}_{2}}{d t}\right)
\end{aligned}
$$

$25 \mathrm{pH}$ values were then calculated by applying the $\left[\mathrm{H}^{+}\right]$trend to the 2010 mean value $\left(\left[\overline{\mathrm{H}^{+}}\right]\right)$in increments of 1 year from $t=0$ to 9 to simulate one decade.

$$
\mathrm{pH}(t)=-\log \left(\left[\overline{\mathrm{H}^{+}}\right]+(t-4.5) \times\left(\frac{d\left[\mathrm{H}^{+}\right]}{d t}\right)\right)
$$

A simple linear regression was then applied to the $\mathrm{pH}$ values resulting from Eq. (3) to estimate the 10-year $\mathrm{pH}$ trend. To estimate $\mathrm{pH}$ trend uncertainty for the two sites from Sutton et al. (2019), the $\mathrm{pH}$ trend was divided by the $\left[\mathrm{H}^{+}\right]$ trend (to determine the local sensitivity of $\mathrm{pH}$ to $\left[\mathrm{H}^{+}\right]$changes) and multiplied by the $\left[\mathrm{H}^{+}\right]$trend uncertainty.

$$
u(\mathrm{pH})=u\left(\frac{d\left[\mathrm{H}^{+}\right]}{d t}\right) \times\left(\left(\frac{d \mathrm{pH}}{d t}\right) \times\left(\frac{d\left[\mathrm{H}^{+}\right]}{d t}\right)^{-1}\right)
$$

$\mathrm{pH}$ trends and uncertainties are presented in Table S1 and Fig. S1. 
Table S1. Mooring and ship-based time-series site names, latitudes, longitudes, sea surface $\mathrm{pH}$ trends, $\mathrm{pH}$ trend uncertainties, $\mathrm{pH}$ trend observing periods, and the literature references from which these data were obtained. Also included are estimates of the 2010 mean $\mathrm{pH}$ value for each site and the literature references from which these mean values were obtained. All values are at in situ temperature on the total hydrogen ion scale.

\begin{tabular}{|c|c|c|c|c|c|c|c|c|}
\hline Location & $\begin{array}{l}\text { Lat. } \\
\left({ }^{0} \mathrm{~N}\right)\end{array}$ & $\begin{array}{l}\text { Lon. } \\
\left({ }^{0} \mathbf{E}\right)\end{array}$ & $\begin{array}{c}\text { pH } \\
\text { Trend } \\
\left(\mathbf{y r}^{-1}\right)\end{array}$ & $\begin{array}{c}\text { pH Trend } \\
\text { Uncertainty } \\
\left(\mathrm{yr}^{-1}\right)\end{array}$ & $\begin{array}{c}\text { pH Trend } \\
\text { Observing } \\
\text { Period }\end{array}$ & $\begin{array}{l}\text { pH Trend } \\
\text { Reference }\end{array}$ & $\begin{array}{l}\text { Mean } \\
\text { pH in } \\
2010\end{array}$ & $\begin{array}{l}\text { Mean pH } \\
\text { Reference }\end{array}$ \\
\hline $170^{\circ} \mathrm{W}$ & 0.00 & 190.00 & -0.0010 & 0.0004 & 2005-2011 & 1 & 7.996 & $4 a$ \\
\hline $155^{\circ} \mathrm{W}$ & 0.00 & 205.00 & -0.0022 & 0.0003 & $1998-2011$ & 1 & 7.998 & $4 a$ \\
\hline $140^{\circ} \mathrm{W}$ & 0.00 & 220.00 & -0.0018 & 0.0004 & 2004-2011 & 1 & 7.981 & $4 a$ \\
\hline $125^{\circ} \mathrm{W}$ & 0.00 & 235.00 & -0.0026 & 0.0005 & $2004-2011$ & 1 & 7.973 & $4 a$ \\
\hline Iceland & 68.00 & 347.34 & -0.0014 & 0.0005 & 1983-2009 & 2 & 8.110 & $4 \mathrm{~b}$ \\
\hline Irminger & 64.30 & 332.00 & -0.0026 & 0.0006 & $1983-2005$ & 2 & 8.081 & $4 \mathrm{~b}$ \\
\hline BATS & 32.00 & 296.00 & -0.0017 & 0.0001 & $1983-2012$ & 2 & 8.085 & $4 \mathrm{~b}$ \\
\hline ESTOC & 29.04 & 344.50 & -0.0018 & 0.0002 & $1995-2012$ & 2 & 8.074 & $4 a$ \\
\hline CARIACO & 10.50 & 295.32 & -0.0025 & 0.0004 & $1995-2012$ & 2 & 8.047 & $4 a$ \\
\hline Munida & -45.70 & 171.50 & -0.0013 & 0.0004 & $1998-2012$ & 2 & 8.090 & $4 a$ \\
\hline DP N (R1) & -57.00 & 296.00 & -0.0019 & 0.0002 & $2002-2015$ & 3 & 8.061 & 3 \\
\hline DP S (R4) & -61.50 & 298.00 & -0.0015 & 0.0003 & $2002-2015$ & 3 & 8.060 & 3 \\
\hline WHOTS & 22.67 & 202.02 & -0.0019 & 0.0003 & $2004-2014$ & 5 & 8.056 & $4 a$ \\
\hline Stratus & -19.70 & 274.40 & -0.0015 & 0.0003 & $2006-2015$ & 5 & 8.062 & $4 a$ \\
\hline ALOHA/HOT & 22.75 & 202.00 & -0.0017 & 0.0001 & $1988-2012$ & 6 & 8.077 & 6 \\
\hline
\end{tabular}

${ }^{1}$ Sutton et al., 2014

${ }^{2}$ Bates et al., 2014

${ }^{3}$ Munro et al., 2015: Mean $\mathrm{pH}$ values were adjusted to the year 2010 using the $\mathrm{pH}$ trends and observing periods.

${ }^{4}$ Fassbender et al., 2017: 2010 centered (a) SOCAT-v4 global climatology and (b) time-series site climatologies.

${ }^{5}$ Sutton et al., 2019: $p \mathrm{CO}_{2}$ trends and uncertainties were converted to $\mathrm{pH}$ trends and uncertainties using the calculations outlined in Text S2 of this study

$45 \quad{ }^{6}$ Dore et al., 2014: $\mathrm{pH}$ values were calculated from $\left[\mathrm{H}^{+}\right]$information provided in the manuscript. The mean $\left[\mathrm{H}^{+}\right]$ value was adjusted to the year 2010 using the $\left[\mathrm{H}^{+}\right]$trend and observing period. 
Table S2. Mooring and ship-based time-series site names, latitudes, longitudes, and literature references from which these data were obtained. The right six columns show $\mathrm{pH}$ and $\left[\mathrm{H}^{+}\right]$values calculated from GFDL ESM2M model output for the combined historical and RCP8.5 experiments for total alkalinity, $p \mathrm{CO}_{2}$, silicate, phosphate, salinity, and temperature using the MATLAB program CO2SYS version 1.1 (van Heuven et al., 2011; Lewis and Wallace, 1998). The dissociation constants of Mehrbach et al. [1973] as refit by Dickson and Millero [1987], the hydrogen sulfate dissociation constant of Dickson et al. [1990], and the boron-to-chlorinity ratio of Uppström [1974] were applied in these calculations, following the approach used for ESM2M. Provided are the average 1950s and 2090s annual mean sea surface $\mathrm{pH}$ and $\left[\mathrm{H}^{+}\right]\left(\mathrm{nmol} \mathrm{kg}{ }^{-1}\right)$ values and the change in decadal average sea surface $\mathrm{pH}$ and $\left[\mathrm{H}^{+}\right]\left(\mathrm{nmol} \mathrm{kg}^{-1}\right)$ seasonal cycle amplitude $(\Delta \mathrm{A})$ between the 1950 s and 2090s. Decadal averages were smoothed with a running mean filter using a four-element, sliding window to reduce the influence of natural variability.

\begin{tabular}{|c|c|c|c|c|c|c|c|c|c|}
\hline Location & $\begin{array}{l}\text { Lat. } \\
\left({ }^{\circ} \mathbf{N}\right)\end{array}$ & $\begin{array}{l}\text { Lon. } \\
\left({ }^{\circ} \mathbf{E}\right)\end{array}$ & Ref. & $\begin{array}{c}\text { 1950s } \\
\text { Annual } \\
\text { Mean } \\
\text { pH }\end{array}$ & $\begin{array}{c}\text { 2090s } \\
\text { Annual } \\
\text { Mean } \\
\text { pH }\end{array}$ & $\begin{array}{c}\text { 2090s- } \\
1950 \mathrm{~s} \\
\Delta \mathrm{A}-\mathrm{pH}\end{array}$ & $\begin{array}{c}\text { 1950s } \\
\text { Annual } \\
\text { Mean }\left[\mathrm{H}^{+}\right] \\
\left(\mathrm{nmol} \mathrm{\mathbf { } \mathrm { g } ^ { - 1 }}\right)\end{array}$ & $\begin{array}{c}\text { 2090s } \\
\text { Annual } \\
\text { Mean }\left[\mathbf{H}^{+}\right] \\
\left(\mathbf{n m o l ~ ~ ^ { - 1 }}\right)\end{array}$ & $\begin{array}{c}\text { 2090s- } \\
1950 \mathrm{~s} \Delta \mathrm{A}- \\
{\left[\mathrm{H}^{+}\right]} \\
\left(\mathrm{nmol} \mathrm{kg}^{-1}\right)\end{array}$ \\
\hline ALAWAI & 21.28 & 202.15 & 1 & 8.154 & 7.770 & -0.013 & 7.022 & 16.992 & 0.5987 \\
\hline ВОВОА & 14.97 & 89.93 & 1 & 8.122 & 7.770 & 0.008 & 7.562 & 17.008 & 1.121 \\
\hline BTM & 31.50 & 295.80 & 1 & 8.148 & 7.783 & -0.008 & 7.129 & 16.553 & 1.710 \\
\hline CAPEELIZABETH & 47.35 & 235.27 & 1 & 8.285 & 7.829 & -0.083 & 5.263 & 14.916 & 2.185 \\
\hline CCE1 & 33.50 & 237.49 & 1 & 8.121 & 7.740 & -0.031 & 7.598 & 18.243 & 1.018 \\
\hline CCE2 & 33.48 & 239.19 & 1 & 8.134 & 7.756 & -0.020 & 7.384 & 17.585 & 1.649 \\
\hline CHABA & 47.97 & 234.63 & 1 & 8.214 & 7.752 & -0.066 & 6.148 & 17.750 & 1.048 \\
\hline CHEECAROCKS & 24.91 & 279.38 & 1 & 8.127 & 7.783 & -0.008 & 7.480 & 16.497 & 1.251 \\
\hline CHUUK & 7.46 & 151.90 & 1 & 8.116 & 7.761 & 0.008 & 7.650 & 17.335 & 0.786 \\
\hline COASTALMS & 30.00 & 271.40 & 1 & 8.219 & 7.828 & -0.032 & 6.113 & 14.972 & 2.422 \\
\hline CRESCENTREEF & 32.40 & 295.21 & 1 & 8.153 & 7.784 & -0.004 & 7.048 & 16.479 & 1.603 \\
\hline CRIMP1 & 21.43 & 202.21 & 1 & 8.154 & 7.770 & -0.013 & 7.022 & 16.992 & 0.599 \\
\hline CRIMP2 & 21.46 & 202.20 & 1 & 8.154 & 7.770 & -0.013 & 7.022 & 16.992 & 0.599 \\
\hline GAKOA & 59.85 & 210.50 & 1 & 8.189 & 7.736 & -0.059 & 6.502 & 18.371 & 0.383 \\
\hline GRAYSREEF & 31.40 & 279.13 & 1 & 8.148 & 7.792 & -0.005 & 7.138 & 16.200 & 1.893 \\
\hline GULFOFMAINE & 43.02 & 289.46 & 1 & 8.139 & 7.774 & -0.004 & 7.275 & 16.835 & 1.202 \\
\hline HOGREEF & 32.46 & 295.17 & 1 & 8.153 & 7.784 & -0.004 & 7.048 & 16.479 & 1.603 \\
\hline ICELAND & 68.00 & 347.30 & 1 & 8.219 & 7.753 & -0.038 & 6.063 & 17.751 & 1.653 \\
\hline JKEO & 37.93 & 146.52 & 1 & 8.168 & 7.783 & -0.005 & 6.797 & 16.503 & 1.265 \\
\hline KANEOHE & 21.48 & 202.22 & 1 & 8.154 & 7.770 & -0.013 & 7.022 & 16.992 & 0.599 \\
\hline KEO & 32.28 & 144.58 & 1 & 8.162 & 7.772 & -0.014 & 6.915 & 16.950 & 1.701 \\
\hline KILONALU & 21.29 & 202.14 & 1 & 8.154 & 7.770 & -0.013 & 7.022 & 16.992 & 0.599 \\
\hline KODIAK & 57.70 & 207.69 & 1 & 8.154 & 7.732 & -0.011 & 7.029 & 18.564 & 1.091 \\
\hline LAPARGUERA & 17.95 & 292.95 & 1 & 8.128 & 7.778 & -0.007 & 7.447 & 16.672 & 0.665 \\
\hline M2 & 56.51 & 195.96 & 1 & 8.146 & 7.727 & -0.006 & 7.161 & 18.782 & 1.449 \\
\hline NH10 & 44.90 & 235.22 & 1 & 8.163 & 7.750 & -0.047 & 6.908 & 17.795 & 0.638 \\
\hline PAPA & 50.13 & 215.16 & 1 & 8.145 & 7.732 & -0.009 & 7.179 & 18.533 & 1.045 \\
\hline SEAK & 56.26 & 225.33 & 1 & 8.219 & 7.789 & 0.006 & 6.046 & 16.281 & 1.808 \\
\hline SOFS & -46.80 & 142.00 & 1 & 8.143 & 7.757 & 0.008 & 7.192 & 17.509 & 1.167 \\
\hline STRATUS & -19.70 & 274.40 & 1 & 8.133 & 7.778 & -0.010 & 7.370 & 16.677 & 0.523 \\
\hline TAO110W & 0.00 & 250.07 & 1 & 8.047 & 7.739 & -0.015 & 8.984 & 18.254 & 0.054 \\
\hline TAO125W & -0.18 & 235.59 & 1 & 8.059 & 7.746 & -0.013 & 8.738 & 17.964 & -0.015 \\
\hline
\end{tabular}




\begin{tabular}{|c|c|c|c|c|c|c|c|c|c|}
\hline Location & $\begin{array}{l}\text { Lat. } \\
\left({ }^{\circ} \mathbf{N}\right)\end{array}$ & $\begin{array}{l}\text { Lon. } \\
\left({ }^{\circ} \mathbf{E}\right)\end{array}$ & Ref. & $\begin{array}{c}\text { 1950s } \\
\text { Annual } \\
\text { Mean } \\
\text { pH }\end{array}$ & $\begin{array}{c}2090 s \\
\text { Annual } \\
\text { Mean } \\
\text { pH }\end{array}$ & $\begin{array}{c}\text { 2090s- } \\
1950 \text { s } \\
\Delta A-p H\end{array}$ & $\begin{array}{c}\text { 1950s } \\
\text { Annual } \\
\text { Mean }\left[\mathbf{H}^{+}\right] \\
\left(\mathbf{n m o l} \mathbf{k g}^{-1}\right)\end{array}$ & $\begin{array}{c}\text { 2090s } \\
\text { Annual } \\
\text { Mean }\left[\mathbf{H}^{+}\right] \\
\left(\mathbf{n m o l ~ ~ k ^ { - 1 } )}\right.\end{array}$ & $\begin{array}{c}2090 \mathrm{~s}- \\
1950 \mathrm{~s} \Delta \mathrm{A}- \\
{\left[\mathrm{H}^{+}\right]} \\
\left(\mathrm{nmol} \mathrm{kg}^{-1}\right)\end{array}$ \\
\hline TAO140W & 0.00 & 220.13 & 1 & 8.069 & 7.751 & -0.013 & 8.531 & 17.750 & -0.067 \\
\hline TAO155W & 0.00 & 205.00 & 1 & 8.079 & 7.755 & -0.016 & 8.342 & 17.577 & -0.170 \\
\hline TAO165E & 0.01 & 164.97 & 1 & 8.097 & 7.760 & -0.011 & 7.999 & 17.378 & 0.050 \\
\hline TAO170W & -0.04 & 189.95 & 1 & 8.087 & 7.758 & -0.015 & 8.184 & 17.472 & -0.143 \\
\hline TAO8S165E & -8.04 & 164.79 & 1 & 8.115 & 7.761 & 0.004 & 7.674 & 17.347 & 0.723 \\
\hline WHOTS & 22.67 & 202.02 & 1 & 8.154 & 7.770 & -0.015 & 7.017 & 17.004 & 0.591 \\
\hline BATS & 32.00 & 296.00 & 2 & 8.148 & 7.783 & -0.008 & 7.129 & 16.553 & 1.710 \\
\hline HOT & 22.75 & 202.00 & 2 & 8.154 & 7.769 & -0.016 & 7.030 & 17.028 & 0.573 \\
\hline Iceland & 68.00 & 347.34 & 2 & 8.219 & 7.753 & -0.038 & 6.063 & 17.751 & 1.653 \\
\hline Irminger & 64.30 & 332.00 & 2 & 8.173 & 7.773 & -0.028 & 6.752 & 16.914 & 1.505 \\
\hline Munida & -45.70 & 171.50 & 2 & 8.171 & 7.788 & 0.008 & 6.759 & 16.344 & 1.809 \\
\hline ESTOC & 29.04 & 344.50 & 2 & 8.147 & 7.788 & -0.009 & 7.137 & 16.305 & 0.894 \\
\hline CARIACO & 10.50 & 295.33 & 2 & 8.096 & 7.775 & -0.007 & 8.023 & 16.783 & 0.528 \\
\hline DPN & -57.00 & 296.00 & 3 & 8.134 & 7.747 & -0.014 & 7.341 & 17.920 & 0.165 \\
\hline DPS & -61.50 & 298.00 & 3 & 8.162 & 7.741 & -0.023 & 6.897 & 18.186 & 0.230 \\
\hline
\end{tabular}

${ }^{1}$ Sutton et al., 2019

${ }^{2}$ Bates et al., 2014

${ }^{3}$ Munro et al., 2015

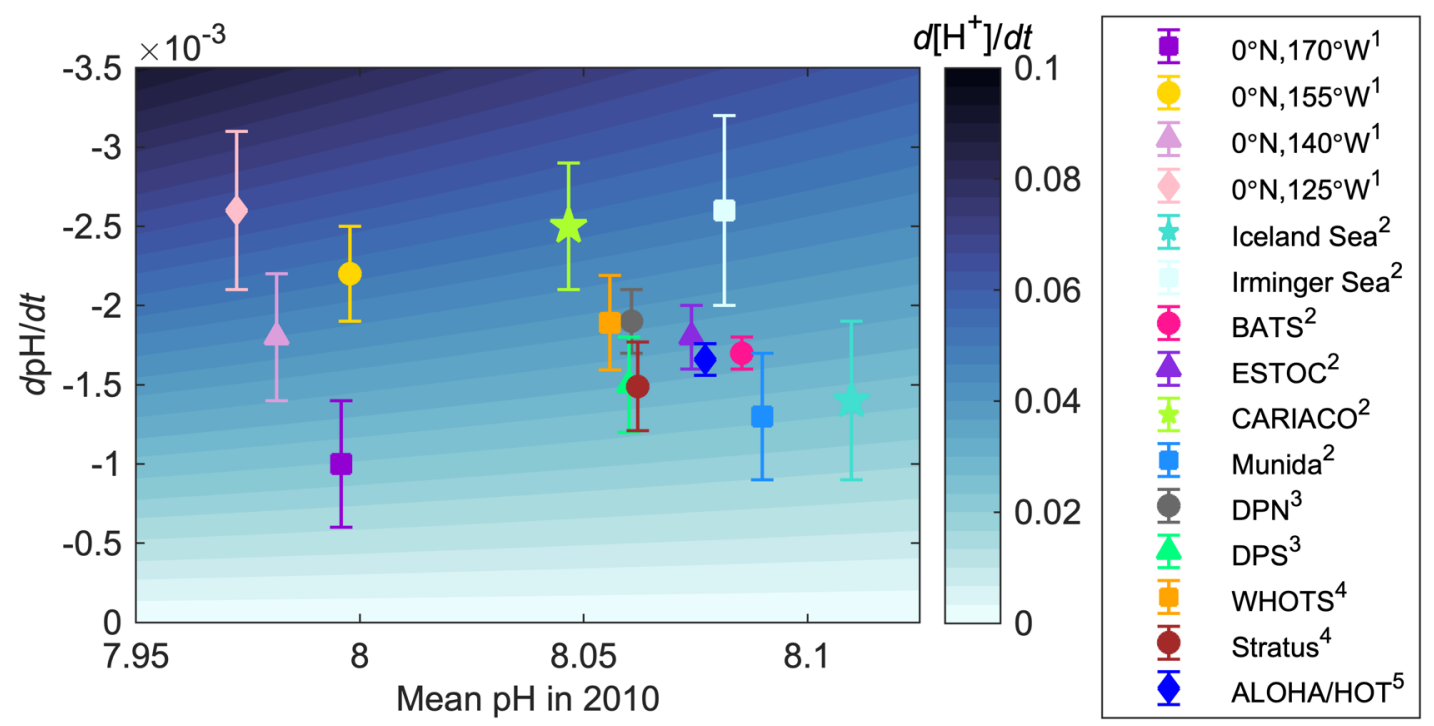

Figure S1. Contour plot showing linearized trends in $\left[\mathrm{H}^{+}\right]\left(\mathrm{nmol} \mathrm{kg}^{-1} \mathrm{yr}^{-1}\right)$ associated with mean (or initial) $\mathrm{pH}$ values referenced to the year 2010 and the corresponding $\mathrm{pH}$ trends. The y axis is reversed so that larger magnitude $\mathrm{pH}$ trends are near the top left corner. Symbols show observed and calculated surface ocean $\mathrm{pH}$ trends and uncertainties (at in situ temperature) at ocean time-series sites, where legend superscripts refer to ${ }^{1}$ Sutton et al. (2014), ${ }^{2}$ Bates et al. (2014), ${ }^{3}$ Munro et al. (2015), ${ }^{4}$ Sutton et al. (2019), and ${ }^{5}$ Dore et al. (2014). These sites include: four equatorial Pacific locations, Iceland Sea, Irminger Sea, Bermuda Atlantic Time-series Study (BATS), European Station for Time series in the Ocean at the Canary Islands (ESTOC), CArbon Retention In A Colored Ocean sites in the North Atlantic (CARIACO), Munida, two sites in the Drake Passage north (DPN) and south (DPS) of the Antarctic Polar Front, the Woods Hole Oceanographic Institution HOT Station (WHOTS), the Stratus site, and the ALOHA/Hawaii Ocean 
Time-series (HOT) site. The Drake Passage sites equate to Regions 1 (DPN) and 4 (DPS) in Munro et al., (2015). Details regarding the determination of mean $\mathrm{pH}$ values referenced to the year 2010 as well as $\mathrm{pH}$ trends and uncertainties for these time-series sites are described in Text S1 and Text S2 and presented in Table S1.
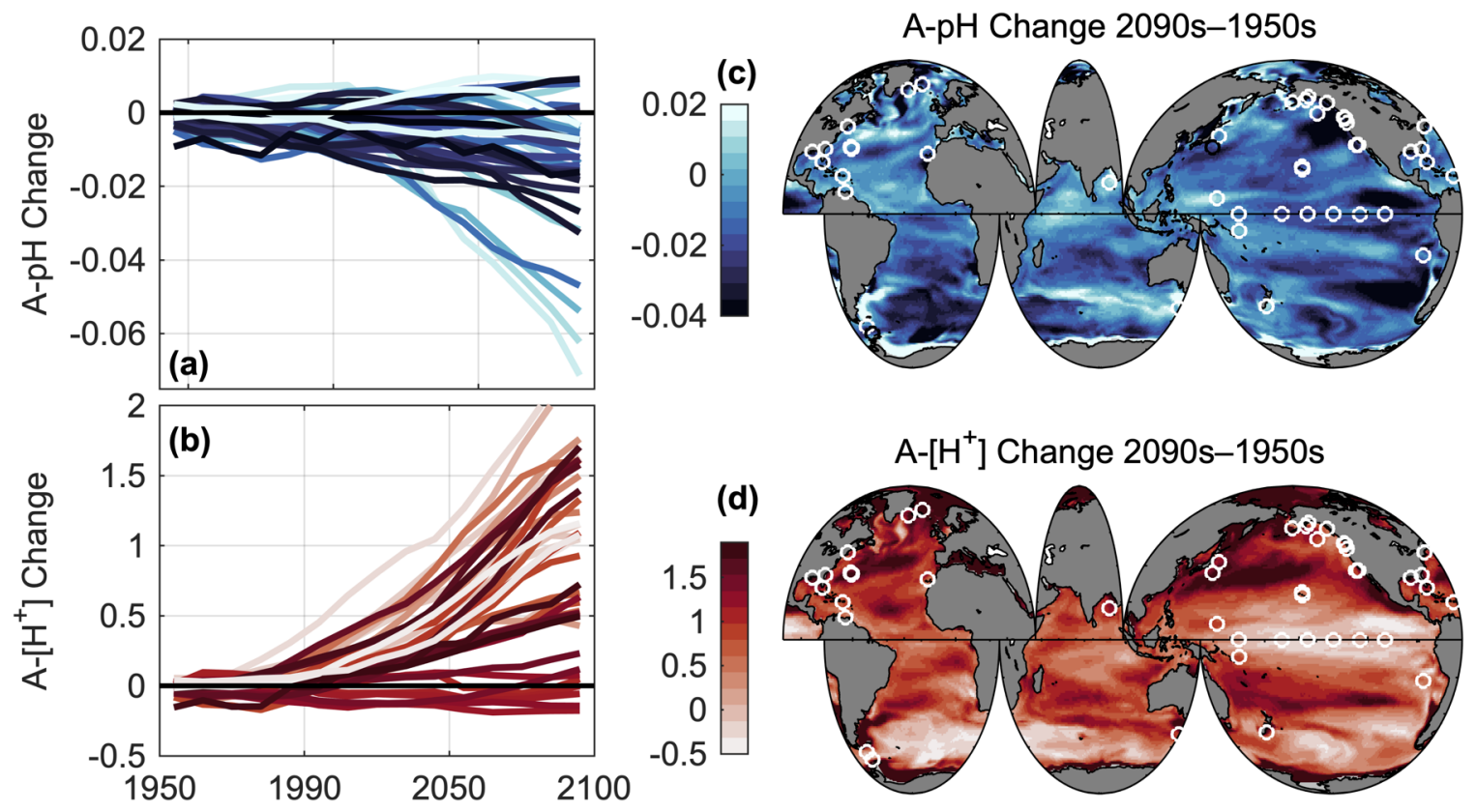

Figure S2. Data used in this figure come from the GFDL ESM2M model for the combined historical and RCP8.5 experiments. Surface ocean seasonal cycle amplitudes (A) were averaged for each decade and smoothed with a running mean filter using a four-element, sliding window. Shown are the decadal changes in (a) A-pH and (b) A- $\left[\mathrm{H}^{+}\right](\mathrm{nmol}$ $\mathrm{kg}^{-1}$ ) relative to the 1950s at model grids corresponding to time-series site locations in ${ }^{1}$ Bates et al. (2014), ${ }^{2}$ Munro et al. (2015), and ${ }^{3}$ Sutton et al. (2019; Table S2). Global maps show the total change in (c) A-pH and (d) A- $\left[\mathrm{H}^{+}\right](\mathrm{nmol}$ $\mathrm{kg}^{-1}$ ) between the 1950s and 2090s. White circles represent the time-series site locations for data presented in a and b. Simulated 1950s and 2090s annual mean $\mathrm{pH}$ and $\left[\mathrm{H}^{+}\right]$values as well as 2090s minus 1950s change in A-pH and A$\left[\mathrm{H}^{+}\right]$at each of the 47 locations are listed in Table S2.

\section{References}

Bakker, D. C. E., Pfeil, B., Landa, C. S., Metzl, N., O’Brien, K. M., Olsen, A., Smith, K., Cosca, C., Harasawa, S., Jones, S. D., Nakaoka, S., Nojiri, Y., Schuster, U., Steinhoff, T., Sweeney, C., Takahashi, T., Tilbrook, B., Wada, C., Wanninkhof, R., Alin, S. R., Balestrini, C. F., Barbero, L., Bates, N. R., Bianchi, A. A., Bonou, F., Boutin, J.,

95 Bozec, Y., Burger, E. F., Cai, W.-J., Castle, R. D., Chen, L., Chierici, M., Currie, K., Evans, W., Featherstone, C., Feely, R. A., Fransson, A., Goyet, C., Greenwood, N., Gregor, L., Hankin, S., Hardman-Mountford, N. J., Harlay, J., Hauck, J., Hoppema, M., Humphreys, M. P., Hunt, C. W., Huss, B., Ibánhez, J. S. P., Johannessen, T., Keeling, R., Kitidis, V., Körtzinger, A., Kozyr, A., Krasakopoulou, E., Kuwata, A., Landschützer, P., Lauvset, S. K., Lefèvre, N., Lo Monaco, C., Manke, A., Mathis, J. T., Merlivat, L., Millero, F. J., Monteiro, P. M. S., Munro, D. R., Murata, A.,

100 Newberger, T., Omar, A. M., Ono, T., Paterson, K., Pearce, D., Pierrot, D., Robbins, L. L., Saito, S., Salisbury, J., Schlitzer, R., Schneider, B., Schweitzer, R., Sieger, R., Skjelvan, I., Sullivan, K. F., Sutherland, S. C., Sutton, A. J., Tadokoro, K., Telszewski, M., Tuma, M., van Heuven, S. M. A. C., Vandemark, D., Ward, B., Watson, A. J. and $\mathrm{Xu}$, S.: A multi-decade record of high-quality $f \mathrm{CO}_{2}$ data in version 3 of the Surface Ocean $\mathrm{CO}_{2}$ Atlas (SOCAT) Dorothee, Earth Syst. Sci. Data, 8(2), 383-413, doi:10.5194/essd-8-383-2016, 2016.

105 Bates, N., Astor, Y., Church, M. J., Currie, K., Dore, J., Gonaález-Dávila, M., Lorenzoni, L., Muller-Karger, F., Olafsson, J. and Santana-Casiano, J. M.: A time-series view of changing ocean chemistry due to ocean uptake of anthropogenic $\mathrm{CO}_{2}$ and ocean acidification, Oceanography, 27(1), 126-141, doi:10.5670/oceanog.2014.16, 2014.

Dickson, A. G., Wesolowski, D. J., Palmer, D. A. and Mesmer, R. E.: Dissociation constant of bisulfate ion in aqueous sodium chloride solutions to $250^{\circ} \mathrm{C}$, J. Phys. Chem., 94(20), 7978-7985, doi:10.1021/j100383a042, 1990. 
110 Dickson, A. G. G. and Millero, F. J. J.: A comparison of the equilibrium constants for the dissociation of carbonic acid in seawater media, Deep Sea Res. Part A. Oceanogr. Res. Pap., 34(10), 1733-1743, doi:10.1016/01980149(87)90021-5, 1987.

Dore, J. E., Church, M. J., Karl, D. M., Sadler, D. W. and Letelier, R. M.: Paired windward and leeward biogeochemical time series reveal consistent surface ocean $\mathrm{CO}_{2}$ trends across the Hawaiian Ridge, Geophys. Res. 115 Lett., 41(18), 6459-6467, doi:10.1002/2014GL060725, 2014.

Fassbender, A. J., Sabine, C. L. and Palevsky, H. I.: Nonuniform ocean acidification and attenuation of the ocean carbon sink, Geophys. Res. Lett., 44(16), 8404-8413, doi:10.1002/2017GL074389, 2017.

Hagens, M. and Middelburg, J. J.: Generalised expressions for the response of $\mathrm{pH}$ to changes in ocean chemistry, Geochim. Cosmochim. Acta, 187, 334-349, doi:10.1016/j.gca.2016.04.012, 2016.

120 van Heuven, S. M. A. C., Pierrot, D., Rae, J. W. B., Lewis, E. and Wallace, D. W. R.: MATLAB program developed for $\mathrm{CO}_{2}$ system calculations, ORNL/CDIAC-105b. Carbon Dioxide Inf. Anal. Center, Oak Ridge Natl. Lab. U.S. Dep. Energy, Oak Ridge, Tennessee, doi:10.3334/CDIAC/otg.CO2SYS_MATLAB_v1.1, 2011.

Lewis, E. and Wallace, D. W. R.: Program developed for $\mathrm{CO}_{2}$ system calculations, Carbon Dioxide Inf. Anal. Center, Oak Ridge Natl. Lab. U.S. Dep. Energy, Oak Ridge, Tennessee. Environ. Sci. Div. Publ. No. 4735 [online]

125 Available from: https://www.nodc.noaa.gov/ocads/oceans/CO2SYS/co2rprt.html (Accessed 2 July 2014), 1998.

Mehrbach, C., Culberson, C. H., Hawley, J. E. and Pytkowicx, R. M.: Measurement of the apparent dissociation constants of carbonic acid in seawater at atmospheric pressure, Limnol. Oceanogr., 18(6), 897-907, doi:10.4319/1o.1973.18.6.0897, 1973.

Munro, D. R., Lovenduski, N. S., Takahashi, T., Stephens, B. B., Newberger, T. and Sweeney, C.: Recent evidence 130 for a strengthening $\mathrm{CO}_{2}$ sink in the Southern Ocean from carbonate system measurements in the Drake Passage (2002-2015), Geophys. Res. Lett., 42(18), 7623-7630, doi:10.1002/2015GL065194, 2015.

Sutton, A. J., Feely, R. A., Sabine, C. L., McPhaden, M. J., Takahashi, T., Chavez, F. P., Friederich, G. E. and Mathis, J. T.: Natural variability and anthropogenic change in equatorial Pacific surface ocean $p \mathrm{CO}_{2}$ and $\mathrm{pH}$, Global Biogeochem. Cycles, 28(2), 131-145, doi:10.1002/2013GB004679, 2014.

135 Sutton, A. J., Feely, R. A., Maenner-Jones, S., Musielwicz, S., Osborne, J., Dietrich, C., Monacci, N., Cross, J., Bott, R., Kozyr, A., Andersson, A. J., Bates, N. R., Cai, W.-J., Cronin, M. F., De Carlo, E. H., Hales, B., Howden, S. D., Lee, C. M., Manzello, D. P., McPhaden, M. J., Meléndez, M., Mickett, J. B., Newton, J. A., Noakes, S. E., Noh, J. H., Olafsdottir, S. R., Salisbury, J. E., Send, U., Trull, T. W., Vandemark, D. C. and Weller, R. A.: Autonomous seawater $p \mathrm{CO}_{2}$ and $\mathrm{pH}$ time series from 40 surface buoys and the emergence of anthropogenic trends, Earth Syst.

140 Sci. Data, 11(1), 421-439, doi:10.5194/essd-11-421-2019, 2019.

Uppström, L. R.: The boron-chlorinity ratio of deep seawater from the Pacific Ocean, Deep. Res. Part I, 21, 161$162,1974$. 